\section{GP65 A CASE REPORT ON NOROVIRUS RELATED URTICARIAL RASH IN PAEDIATRIC PATIENT}

Navdeep Kaur Brar*, Bazlin Ramly, Cathal O'Connor, Juliette Lucey. Waterford University Hospital, Waterford, Ireland

10.1136/archdischild-2019-epa.131

There are several causes of infectious and non-infectious urticaria recognized. Reports described association with streptococcus, mycoplasma pneumoniae, parvovirus B19, enterovirus, hepatitis A or B and plasmodium falciparum and urticaria. Norovirus is a recognised albeit rare cause of urticaria. Leiste A et al. have published two cases identified on Norovirusrelated urticaria in adult patients. There are no cases yet described in paediatric population on Norovirus related urticaria. Following is the unique case of norovirus related urticaria in paediatric patient.

A two year old, male patient was admitted to the paediatric ward with a sudden episode of generalised florid rash proceeding with multiple episodes of diarrhoea and vomiting. There was no involvement of mucous membranes and joints. The rash was erythematous, with widespread wheals spreading all over the body associated with intensive pruritus which left excoriation marks. The rash and pruritus was subsided with a course of antihistamines. There was no pyrexia documented during the episode and there was no history of atopies. He attends crèche and there was recent acute gastroenteritis in a crèche attendee. On physical examination, the only positive finding was mild to moderate dehydration with no evidence of shock thus intravenous fluid was commenced. His blood investigations done included full blood count, C-reactive protein, renal profile, blood cultures, extended viral panel on nasal swab were normal though stool culture was positive for norovirus. The rash was subsided completely within few hours. Patient was discharged home the following day.

Discussion Noroviruses are genetically diverse group of singlestranded positive -sense RNA, non-enveloped virus belonging to the family Caliciviridea. It primarily causes gastrointestinal symptoms. Patients usually feel very unwell with nausea, vomiting and diarrhoea. A headache and fever may occur. Norovirus is very contagious. It is mainly transmitted by direct person to person contact or through contaminated food/water. It is found in all age group and more prevalent in the winter months. Complications may include dehydration. The incubation period is up to 3 days. There were two cases described previously in adult patient which had an association with urticaria. Comparing the reports from adult's patients, the evidence strongly suggests association between norovirus and urticaria. There is need for further studies and case reports to justify the association.

\section{GP66 FATAL CASE OF MULTIPLE EMBRYONAL TUMORS IN A CHILD WITH BECKWITH-WIEDEMANN SYNDROME}

Anna Stanzelova*, Vladimira Vojtova, Vladimira Jerabkova, Adela Misove, Josef Malis, Karel Svojgr. Department of Paediatric Haemato-Oncology, University Hospital Motol, Prague, Czech Republic

10.1136/archdischild-2019-epa.132

Beckwith-Wiedemann syndrome (BWS) is the most common genetic cause of overgrowth in early childhood and also the most common syndrome caused by the loss of imprinting.
Depending on the underlying molecular genetics, it can increase the risk of development of embryonal tumours by up to $20 \%$. Children with BWS benefit from radiographic and biochemical screening. In case of early detection of malignancy they are promptly treated with similar or better survival than general population. Multiple concomitant tumours remain rare.

We report a case of a 4-year-old boy diagnosed with BWS at birth. This was based on his clinical features and subsequently confirmed genetically as paternal uniparental disomy (11p15.5). His first malignancy was a hepatoblastoma at birth, treated by surgical resection and chemotherapy. Four months later, a suspected left sided nephroblastoma was identified and left sided nephrectomy was performed. Histology revealed nephroblastomosis. 2 years later, a nephroblastoma in the upper pole of right kidney was described. It responded well to chemotherapy and was resected using nephron sparing surgery technique. Three months into adjuvant chemotherapy, another tumor developed in the lower lobe of right kidney. This progressed despite an intensified chemotherapeutic treatment and a nephrectomy was carried out rendering the patient anephric. Radiation to the abdomen with a boost to the site of the right kidney was offered to the boy, while he was on dialysis. Within 2 months of completion of radiation, a new right sided mass was described on follow-up abdominal imaging. At that point all further oncologic treatment was deemed futile and the boy passed away 6 weeks later.

A review of our 30 year patient registry revealed 295 patients diagnosed with nephroblastoma between July 1988 and December 2018. There were 7 children with clinically suspected Beckwith-Widemann syndrome, 2 of those confirmed genetically. All but one were successfully treated without relapse, synchronous or metachronous malignancy.

Unlike other tumour predisposition syndromes, the increased risk of malignancy in BWS reduces sharply after 8 years of life. Synchronous or metachronous malignancy in this population remain exceedingly rare, making our patient an unfortunate exception. Patients with BWS rendered anephric following bilateral nephroblastoma resection have to remain in complete remission for 2 years post completion of oncological therapy before being considered for a renal transplant. Should they remain without evidence of disease, they are good candidates for kidney transplant, with similar rates of survival and graft rejection to other transplantees.

\section{GP67 A CASE REPORT OF AN INFANT WITH GIANT CONGENITAL MELANOCYTES NAEVUS WITH NEUROCUTANEOUS MELANOSIS}

${ }^{1}$ Claire Thompson*, ${ }^{1}$ Kerrie Hennigan, ${ }^{1}$ Siobhan McCormack, ${ }^{2}$ Niazy Al-Assaf, ${ }^{1}$ Rizwan Khan. 'Limerick University Maternity Hospital, Limerick, Ireland; ${ }^{2}$ Limerick University Maternity Hospital, Limerick, Ireland

\subsection{6/archdischild-2019-epa.133}

Background A term infant with features of Giant Congenital Melanocytic Naevus (GCMN) with Neurocutaneous Melanosis (NCM).

Case report A term, female infant, with an uncomplicated antenatal course, of non-consanguineous parents, was noted at birth to have extensive pigmentation of the body.

Examination demonstrated hyperpigmented, rugged, plaquelike lesions in a bathing suit distribution. Within the 